\title{
Numerical studies on the interactions between a flexible plate and incoming flow
}

\author{
Jie Weng ${ }^{1}$, Songjuan $\mathrm{Zhu}^{1}$, Yubin $\mathrm{Cai}^{1}$, Haiqing Xiong ${ }^{1}$ and JianxinHu ${ }^{2, a}$ \\ ${ }^{1}$ Hangzhou Institute of Test and Calibration for Quality and Technology Supervision, Hangzhou 310019, China \\ ${ }^{2}$ Dept. of Naval Architecture, Ocean and Marine Engineering, University of Strathclyde, GlasgowG4 OLZ, United \\ Kingdom
}

\begin{abstract}
The linear Euler - Bernoullibeam model is employed for the studies on the interactions between a flexible plate and incoming flow. The flow field was simulated by the immersed-boundary method. It is shown that the plate was inhibited at a relative low Reynolds number. When the Reynolds increases, the plate begins to flap and its amplitude increases with the increasing of Reynolds number. The amplitude is small with a large stiffness coefficient. When the mass ratio becomes large, the inertial effect enhances, and the amplitude of the plate is large. With the same material parameters, different initial trailing edge inclination lead to different flapping amplitudes.
\end{abstract}

Keywords: Euler - Bernoullibeam; fluids structure interactions (FSI); energy harvesting.

\section{Introduction}

Fish fins are often with certain flexibility. However, in previous study, it is always considered as a rigid body, neglecting the elasticity of the material itself. In order to obtain a comprehensive and accurate understanding of the propulsive properties of fish fins in fluid, it is necessary to introduce an elastic structure model to investigate their interaction. In addition, elastic material interacts with fluid is common in nature and industry, e.g. in papermaking industry [1], the palate in human upper respiratory tract [2], the human body the valves of the heart [3] and elastic piezo material for energy harvesting [4]. How to avoid or benefit from the interactions with the fluid could be the key research topic.

There are serval studies reported in the literatures concerning the interaction between elastic material and its surrounding fluid [5-10]. Specially, Argentina and Mahadevan [7] employed a linear theory to represent an oscillating flag, and they concluded that the flag began to oscillate due to the elastic material minimum natural frequency coincides to the frequency of fluid force. Alben and Shelley [8] investigated the nonlinear oscillations of an elastic thin plate in inviscid fluid, based on inextensible thin elastic plate theory. The bistability and hysteresis phenomena were captured in their simulation.

In this paper, we take into account of the viscous effect by solving the Navier - Stokes(NS) equations, and employed the linear Euler - Bernoullimodel. In the following the corresponding physical model and numerical method are introduced, and then the effects of Reynolds number and the structure parameters are systematically investigated. Finally the conclusion is drawn.

\footnotetext{
${ }^{a}$ Corresponding author: j.hu@strath.ac.uk 


\section{Physical model and numerical method}

\subsection{Physical model}

As shown in Fig. 1,an elastic plate is placed in the center within incoming fluid. The length of the elastic plate is $L$ and the elastic plate thickness is $h=0.03 L$. The left side of computational area is the velocity inlet with velocity $U_{0}$, and the outflow boundary condition is used on the right side. The Reynolds number (Re)is employed and it can be represented as $\operatorname{Re}=U_{0} L / v$, where $v$ is the kinematic viscosity of the fluid.

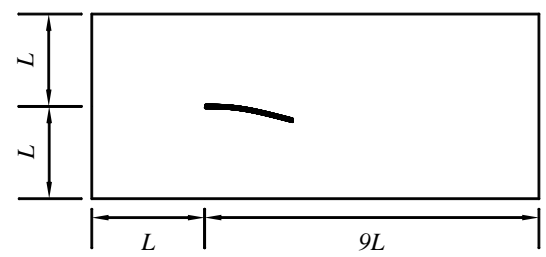

Figure 1. Physical model and computational domain

\subsection{Fluid Solver}

We adopt the incompressible NSequations and it can be non-dimensionized as

$$
\begin{gathered}
\frac{\partial \vec{V}}{\partial t}+(\vec{V} \cdot \nabla) \vec{V}=-\nabla p+\frac{1}{\operatorname{Re}} \nabla^{2} \vec{V}+\vec{F}, \\
\nabla \cdot \vec{V}=0 .
\end{gathered}
$$

Here, $\vec{V}$ and $p$ are corresponding to the velocity and pressure of the fluid. The immersed boundary method is used, coupling with the structured Cartesian grid. By adding an force term into the NSequations, the no-slip boundary condition around the plate can be fulfilled. $\vec{F}(i, j)$ is the additional force, and it only has non-zero value near the wall[4][11].It can be calculated by

$$
\vec{F}^{n+1}=-\overrightarrow{R H S}^{n}+\frac{\vec{U}^{n+1}-\vec{u}^{n}}{\Delta t} .
$$

Here, $\vec{U}^{n+1}$ is the speed of the moving boundary at time $t+\Delta t$, and $\vec{u}^{n}$ is corresponding to the speed at time $t . \overrightarrow{R H S}^{n}$ contains the convection, viscosity and pressure gradient terms in NSequations that can be calculated explicitly.

\subsection{Structure solver}

Concerning the forced oscillation of the elastic plate, the linearized Euler -Bernoullibeam model[12] is introduced.In this model, the effect of fluid shear stress (viscouseffect) on Euler-Bernoulli Beam is ignored and only the fluid. pressure jump cross the plate is considered. Regardless of the material damping coefficient, the governing equation can be non-dimensionized as following ( $\mu$ and $\eta$ are the two control parameters, mass ratio and stiffness coefficient, respectively):

$$
\mu \frac{\partial^{2} w}{\partial t^{2}}+\eta \frac{\partial^{4} w}{\partial x^{4}}=-\Delta p
$$




$$
\mu=\frac{\rho_{s}}{\rho_{f} L}, \eta=\frac{B}{\rho_{f} U_{0}^{2} L^{3}} .
$$

Here, $t, x$ and ware the dimensionless time, length coordinates and the transverse displacement of beam, respectively. $B$ is the stiffness coefficient which can be derived as $B=E h^{3} /\left[12\left(1-v_{s}^{2}\right)\right]$ where $E$ and $v_{s}$ are the elastic modulus and the Poisson ratio respectively. In the simulation, one end of the elastic plate is fixed, and the other end can swing freely. Their boundary conditions are

$$
\begin{array}{r}
w(0, t)=0,\left.\frac{\partial w}{\partial x}\right|_{x=0}=0, \\
\left.\frac{\partial^{2} w}{\partial x^{2}}\right|_{x=1}=0,\left.\frac{\partial^{3} w}{\partial x^{3}}\right|_{x=1}=0 .
\end{array}
$$

The finite difference method is used to explicitly discretize the governing equation of the elastic plate.

\section{Effect of Re number}

For incompressible viscous fluid mechanics problems, the magnitude of Re number reflects the ratio of inertia force to viscous force, which plays an important role in flow pattern and velocity distribution, etc. In this section, the Re number effect is investigated. Currently, the range of Re number is 50 $\sim 250$. In order to stimulate the vibration of the plate, there is an initial incline angle between the elastic plate and the horizontal direction. Consequently, the horizontal displacement of the elastic plate are $w(x)=\zeta x$.

It can be seen in the Fig. 2 that, when $\mathrm{Re}=50$, elastic plate stop to oscillate after its initial fluctuation. When Re number becomes large, displacement of trailing edge start to oscillate periodic with constant amplitude. When $\mathrm{Re}=100$, the oscillating amplitude is smaller than that with Re number 200 and 250.

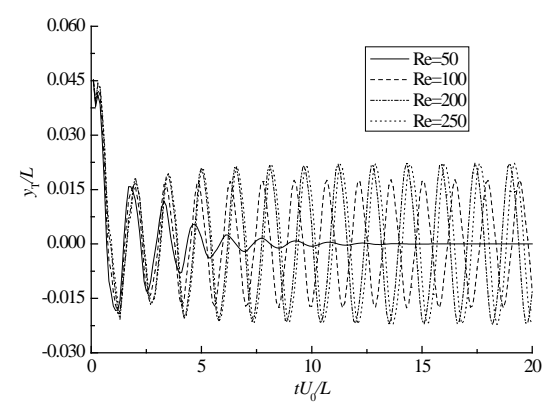

Figure 2.Time history of trailing edge displacement with $\mu=0.5, \eta=0.025$ and $\zeta=0.05$.

It is easy to understand that when the Re number is small, the fluid viscous effect is significant, inhibiting the transverse oscillation of the elastic plate. Figure 3 gives the snapshots of the flow field around the elastic plate. When the Re is 50 , the elastic plate tends to be stationary, and the around vorticity distribution is symmetric. As the Re number increases, the elastic plate starts to oscillate. As shown in Fig. 3, when the Re number is 100 and 200, the vorticities in the wake become instable, and the symmetry distribution is destroyed. 

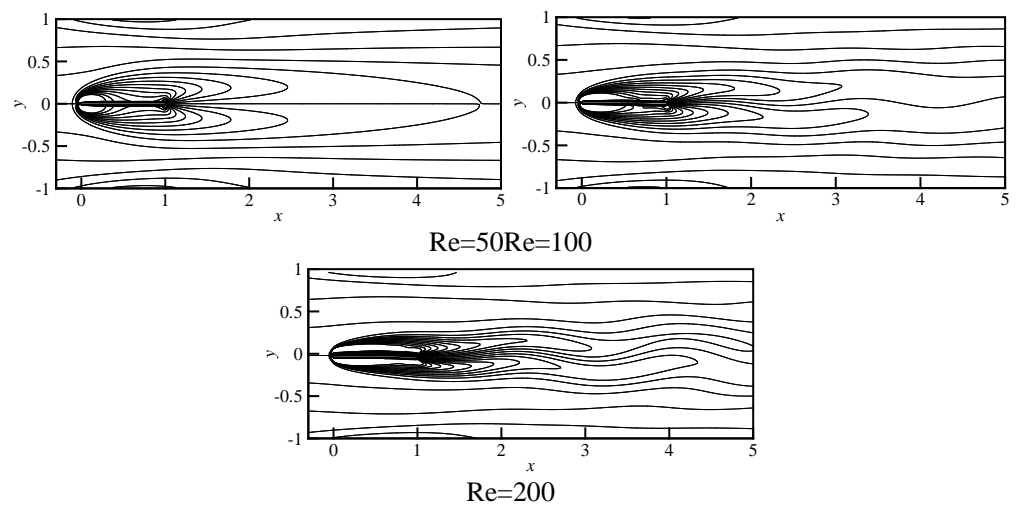

Figure 3.Vorticityfield around elastic plate with different Re number.

\section{Effect of structure properties}

\subsection{Influence of dimensionless parameters $\eta$}

Figure 4 shows the results of plate oscillation behaviors with different stiffness.Two series of cases are mainly considered with fixed density ration of $\mu=0.5$ and $\mu=1.0$, and the variation range of the stiffness coefficients is $\eta=0.025 \sim 1.0$.
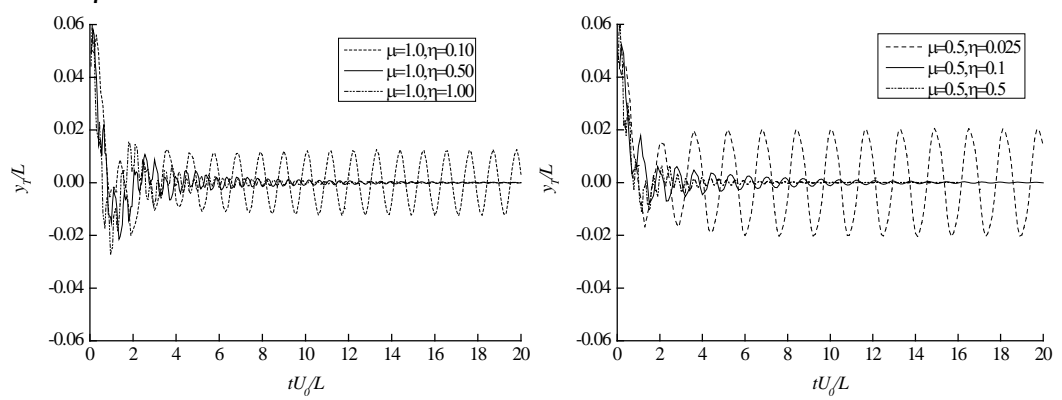

Figure 4.Time history of trailing edge displacement with different stiffness coefficient.

When the stiffness is small, the elastic plate is activated after the initial disturbance, as shown inFig. 4 (left) under condition, $\mu=0.5$ and $\eta=0.025$ and Fig. 4 (right) under condition, $\mu=1.0$ and $\eta=0.10$.For cases with the same density, with the increase of stiffness, the amplitude of plate trailing edge became smaller. According to the physical meaning of stiffness coefficient, the greater the stiffness, the stronger ability to overcome bending effect. According to the results in our simulation, the effect of stiffness coefficient on the oscillation behavior of elastic plate satisfies its physical meaning of the stiffness coefficient.

\subsection{Influence of dimensionless parameters $\mu$}

Density ratio represents plate density over fluid density. Corresponding simulation results are given in Fig. 5. Two groups of parameters are chosen, with the stiffness, $\eta=0.1$ and $\eta=0.5$, and the density ratio varies $\mu=0.5 \sim 2.0$. 

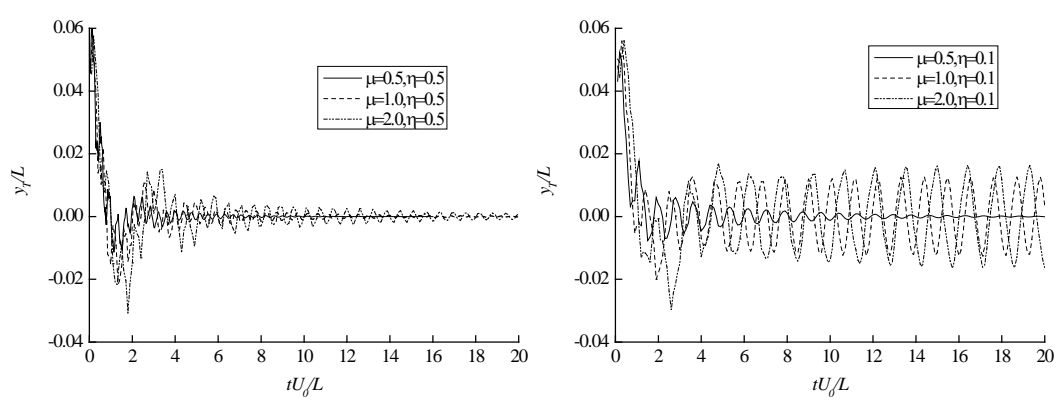

Figure 5.Timehistory of trailing edge displacement with different mass ratio coefficient.

With the same stiffness, when the density ratio is small, the steady amplitude of the trailing edge is small. For cases in the right subfigure, due to large stiffness, the amplitude of the trailing edge is close to zero. Large density ratio causes large inertial effect and hence results large oscillating amplitude.

In addition, the oscillating period of the elastic plate changes for different density ratios, stiffness and Re number. Therefore, this period is determined by the influences of material properties and flow conditions.

\subsection{Influence of trailing edge initial displacement $\zeta$}

In order to stimulate the motion of the elastic plate, the plate is placed with an inclined angle at the initial stage. In this subsection, we investigate the magnitude effect of the initial inclined angle. Two sets of parameters are chosen as $\mu=0.5$ and $\eta=0.025$ for the left subfigure of Fig. 6 and $\mu=2.0$ and $\eta=0.1$ for the right, and there are three different initial displacement $\zeta=0.02,0.05$ and 0.10 .

For the results in the left subfigure, when the initial displacement is small, $\zeta=0.02$ and 0.05 , the free end has the similar small oscillating amplitude, while for that in the right one, when the initial displacement is large, $\zeta=0.05$ and 0.10 , the free end has the similar large oscillating amplitude. The inconsistent results could be the bi-stability phenomenon caused by the nonlinear nature.
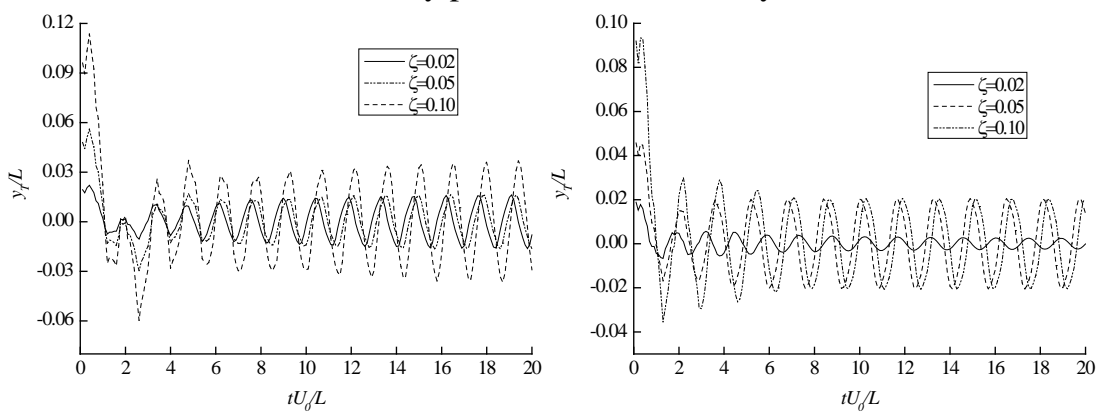

Figure 6. Different initial trailing edge displacement leads to different flapping behaviors of the plate.

\section{Concluding remarks}

Fish swimming in nature has the advantages of high speed, high efficiency, etc., and hence its propulsion mechanism study is a hot spot in the biomimetic research of fluid mechanics. The fins are regarded as rigid in the past, neglecting its flexibility. In this paper, the linearized Euler - Bernoullibeam model is introduced, and its interaction with the flow field is studied, which may provide certain theoretical guidance for the mechanism of the flexible fin propulsion.

This paper mainly studied the effects of Re number and the elastic material parameters, i.e. material stiffness, density ratio and initial displacement. Current study find that when the Re number is small, the motion of the elastic plate is restrained. With the increase of Re number, the elastic plate begins to 
oscillate, and the larger Re number, the larger oscillating amplitude. When the plate stiffness coefficient is greater than 0.5 , the motion of the elastic plate is restrained, tend to be stationary. Under the same stiffness, the greater density ratio, the greater inertial effect of the elastic plate, and the greater oscillation amplitude as well. In addition, when the initial displacement of the plate trailing edge is different, its steady oscillating amplitude also changes. The two-dimension simulation is conducted in current study with neglect of the fluid motion in the third direction, and therefore, a three-dimension simulation is required in the future.

\section{References}

1. Y. Watanabe, S. Suzuki, M. Sugihara and Y. Suroka, An experimental study of paper flutter, J. Fluids Struct., 16, 529 (2002).

2. T. Balint and A. Lucey, Instability of a cantilevered flexible plate in viscous channel flow, $J$. Fluids Struct., 20, 893 (2005).

3. R. van Loon, P. Anderson and F. van de Vosse, A fluid-structure interaction method with solidrigid contact for heart valve dynamics, J. Comput. Phys., 217, 806 (2006).

4. D. Pan, X. Shao, J. Deng and Z. Yu, Simulations of passive oscillation of a flexible plate in the wake of a cylinder by immersed boundary method, Euro. J. Mech. - B/Fluids, 46, 17 (2014).

5. D. Farnell, T. David and D. Barton, Numerical simulation of a filament in a flowing soap film, Int. J. Numer. Meth. Fluids, 44, 313 (2004).

6. J. Eldredge, Dynamically coupled fluid-body interactions in vorticity-based numerical simulations, J. Comput. Phys., 227, 9170 (2008).

7. M. Argentina and L. Mahadevan, Fluid-flow-induced flutter of a flag, Proc. Natl. Acad. Sci. U.S.A., 102, 1829 (2005).

8. S. Alben and M. Shelley, Flapping states of a flag in an inviscid fluid: bistability and the transition to chaos, Phys. Rev. Lett., 100, 074301 (2008).

9. M. Shelley, N. Vandenberghe and J. Zhang, Heavy flags undergo spontaneous oscillation in flowing water, Phys. Rev. Lett., 94, 094302 (2005).

10. Z. Yu, A DLM/FD method for fluid/flexible-body interactions, J. Comput. Phys., 207, 1 (2005).

11. D. Pan, J. Deng, X. Shao and Z. Liu, On the propulsive performance of tandem flapping wings with a modified immersed boundary method, Int. J. Comput. Meth.,13, 1650025 (2016).

12. S. Timoshenko, History of Strength of Materials, With a Brief Account of the History of Theory of Elasticity and Theory of Structures, McGraw-Hill, New York, 1953. 